\title{
Emergent Thrombectomy in a Neonate with an Upper Extremity Arterial Thrombus
}

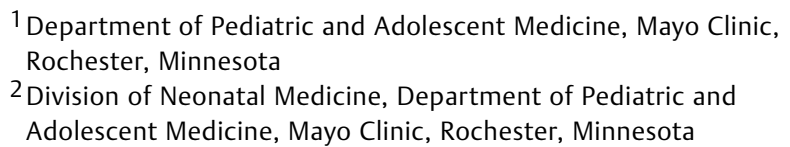 \\ Am J Perinatol Rep 2014;4:41-44.
}

Timothy J. B. Ulrich, MD ${ }^{1}$ Marc A. Ellsworth, MD ${ }^{2}$ Tara R. Lang, MD²

Address for correspondence Timothy J. B. Ulrich, MD, Department of Pediatric and Adolescent Medicine, Mayo Clinic, 200 First Street SW, Rochester, MN 55905 (e-mail: ulrich.timothy@mayo.edu).

\begin{abstract}
Case This case report is of a $39^{4 / 7}$ weeks infant who presented at the time of birth with an immobile, cyanotic right upper extremity consistent with ischemia but without evidence of gangrene. Doppler examination identified pulses in the axillary but not the brachial or radial arteries. Extremity arterial ultrasound confirmed the diagnosis of an arterial thrombosis extending from the right axillary artery to the brachial artery bifurcation. An emergent balloon thrombectomy was performed successfully with immediate return of blood flow. Intraoperative ultrasound demonstrated patent axillary and brachial arteries with forward flow. A retroperitoneal ultrasound and limited

Keywords

- neonate

- arterial thrombus

- thrombectomy

- ischemia hypercoagulable workup failed to identify a source of the arterial thrombus. The infant had normal return of function without residual limb effects.

Conclusion Emergent balloon thrombectomy should be heavily considered in neonates with an extremity arterial thrombosis of undeterminable duration both for limb salvage, preserve function, and to prevent long-term growth discordance.
\end{abstract}

Neonates with a clinically significant arterial thrombosis that develops in utero or during delivery are often born with or quickly develop gangrenous changes, thus illustrating the importance of prompt intervention. However, management guidelines for neonatal arterial thrombosis have not been well established. Generally, recombinant tissue plasminogen activator (rt-PA) has been recommended for limb or lifethreatening thrombi as initial management, but thrombectomy has been recognized as an option in certain situations. When an arterial thrombosis occurs perinatally, it is difficult to determine the duration of occlusion, and limb ischemia and loss may be imminent. As such, thrombectomy may be a superior option to promptly and definitively restore flow. We present a case of a term neonate born with pregangrenous changes in the right arm due to an extensive thrombus extending from the right axillary artery to right brachial artery bifurcation who was subsequently managed successfully with a balloon thrombectomy.

\section{Case}

A 4,980 g, large for gestational age (LGA), male infant was born at $39^{4 / 7}$ weeks' gestation to a 29 -year-old gravida 3, para 1 mother. The pregnancy was complicated by gestational diabetes and a unicornuate uterus. The infant was delivered vaginally and had APGAR scores of 8 and 9 at 1 and 5 minutes, respectively. Despite the infant being LGA, there were no delivery complications requiring instrumentation, however, the umbilical cord was noted to be wrapped around the left arm. At the time of birth, infant was noted to have an immobile, cyanotic right arm. An X-ray performed was suspicious for a right humeral fracture. Because of concern for received

November 11, 2013

accepted after revision

December 24, 2013

published online

March 3, 2014
Dol http://dx.doi.org/

10.1055/s-0034-1370355. ISSN 2157-6998.
Copyright (c) 2014 by Thieme Medical Publishers, Inc., 333 Seventh Avenue, New York, NY 10001, USA. Tel: +1(212) 584-4662.
License terms

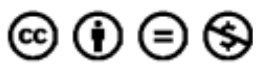


significant vascular compromise the patient was transferred to our institution for further evaluation and management.

Upon arrival to our neonatal intensive care unit (NICU) the orthopedic team consulted. A review of the X-ray and physical evaluation of the patient resolved any concern of humeral fracture. However, the limb continued to be ischemic with absent pulses beyond the clavicle. Doppler examination of the right arm demonstrated obtainable signal in the axillary but not the brachial or radial arteries. A bedside ultrasound showed an acute occlusive thrombus in the right axillary artery extending distally from the clavicle for 4 to $5 \mathrm{~cm}$ to the right brachial artery at the level of the midhumerus (-Fig. $\mathbf{1}$ ).

The patient was given a bolus (75 units/ $\mathrm{kg}$ ) of unfractionated heparin and started on a high dose heparin infusion (28 units $/ \mathrm{kg} / \mathrm{h}$ ). He was taken to the operating room at 10 hours of life for a thrombectomy. Dissection was performed to expose the 3-mm axillary artery. A successful balloon catheter thrombectomy of the axillary and proximal brachial artery was performed. Papaverine was then infused distally with clear demarcation at the antecubital crease. Attention was then turned to the brachial artery bifurcation. Following incision and dissection, forceps were used to remove a small thromboembolus from the right brachial artery bifurcation. The right forearm and hand immediately became pink. Intraoperative ultrasound demonstrated normal arterial flow in the brachial artery proximally and distally (-Fig. 2 ). The patient returned to the NICU and remained on a heparin infusion.

An echocardiogram was performed and was only significant for a patent foramen ovale with a bidirectional shunt. A retroperitoneal ultrasound was performed that did not show evidence of a renal vein thrombus as a possible embolic source. After a brief period of weakness, the infant regained full use of his right arm. A limited hypercoagulation panel yielded normal results. The patient was discharged home without any complications and was followed up by hematology as an outpatient to complete a 3-month course of

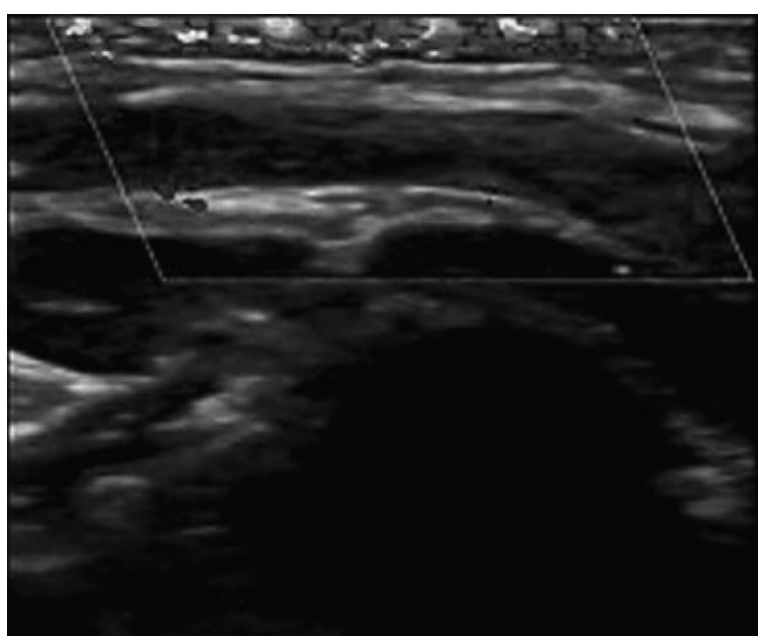

Fig. 1 Preoperative ultrasound of the right brachial artery proximally. Doppler shows obstruction of flow proximally with absent forward flow due to an occlusive thrombus.

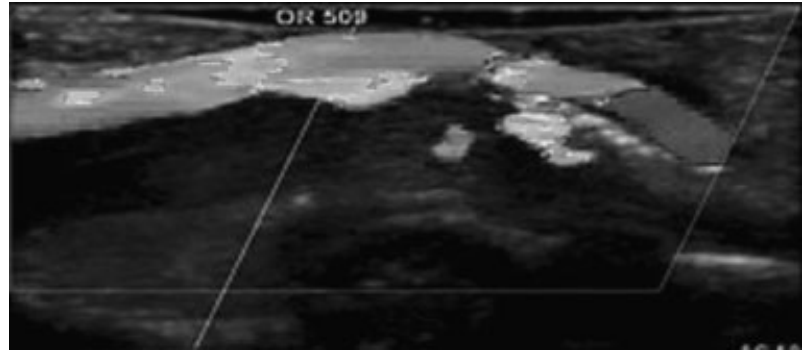

Fig. 2 Postoperative ultrasound of the right brachial artery proximally. Doppler demonstrates forward flow through the previously thrombosed axillary artery.

low-molecular-weight heparin (LMWH) therapy. LMWH therapy was recommended by the hematology service after extrapolation of evidence-based clinical practice guidelines ${ }^{1}$ to best match this patient's clinical scenario.

\section{Discussion}

Clinically significant thrombi are rare in neonates; rarer still are limb and life-threatening arterial thrombi. A prospective report from the Canadian and international registry reported an incidence of clinically apparent thrombosis to be only 2.4 per 1,000 NICU admissions, most of which are due to central venous catheters. ${ }^{2}$ Idiopathic limb ischemia in the neonate has been associated with prematurity, prolonged labor, birth asphyxia, respiratory distress, sepsis, Rhesus disease, congenital anomalies, and maternal diabetes. ${ }^{3}$ Given the infrequency of clinically significant arterial thrombi presenting in the neonatal period, randomized control trials and evidence-based guidelines assessing efficacy of treatment modalities are lacking. Consequently, case reports and case series, as well as extrapolated adult data, have been referenced to help manage these infants. ${ }^{4-10}$

Current therapeutic options for neonatal thrombi include heparinization, fibrinolysis, and thrombectomy. ${ }^{11}$ The decision to treat is approached with great reservation given bleeding risks, especially intraventricular hemorrhage, to which neonates are uniquely susceptible., ${ }^{1,6}$

Neonates with clinically significant arterial thrombi that develop in utero or during the birthing process are often born with or quickly develop gangrenous changes. ${ }^{12}$ Hsi et al notes that over half of the reported cases of neonatal extremity gangrene presented in the first day of life despite a lack of evidence of prolonged in utero thrombosis. ${ }^{13}$ Prompt clearance of the thrombus is thus vital, not only to salvage the limb, but also to preserve limb function and prevent long-term complications including limb growth discordance and exercise-induced claudication. ${ }^{7,8}$

Generally, accepted management of a limb or life-threatening thrombus is the use of fibrinolytics-generally rt-PA-in combination with heparinization. Heparinization alone does not restore blood flow rapidly enough to prevent significant damage. ${ }^{4,5}$ However, fibrinolytics in combination with heparin can also be slow at restoring blood flow to an ischemic limb. Weiner et al report rt-PA infusions require a mean 
duration of therapy of 24.7 hours, with a range of 6 to 39 to treat an arterial thrombosis. ${ }^{10}$ The early onset of gangrenous changes signals the urgency in restoring blood flow, more so when the duration of occlusion is relatively unknown, to prevent long-term limb disability. ${ }^{7}$ Theile and Coombs report a case of a 32-week-gestation neonate who developed right arm ischemia distal to the cubital fossa after placement of a right brachial arterial line. ${ }^{9}$ Microvascular and thrombolytic revascularization was performed. While below the elbow amputation was avoided, portions of the right thumb, index, and ring finger were eventually amputated after 19 hours of ischemia.

In our case, following Doppler and ultrasound evidence of a thrombosed axillary and brachial artery ( - Fig. $\mathbf{1}$ ) as well as a physical examination consistent with limb ischemia, an emergent, uncomplicated balloon thrombectomy with limb salvage was performed. Definite intraoperative return of blood flow was immediately demonstrated (-Fig. 2) and arm function quickly returned without residual defects. As previously stated, the time from birth to clot removal was 10 hours, significantly faster than what would likely be expected with combined fibrinolytic and heparinization therapy.

There are obviously inherent risks to thrombectomy. The vasculature of neonates is both small and friable, predisposing patients to significant complications such as vascular rupture and permanent disruption of blood flow. The risks and benefits of thrombectomy must be considered and compared with those of thrombolysis. When skilled vascular surgery or interventional radiology is available the risks of thrombectomy can be mitigated.

In the absence of evidence-based guidelines for management of infants with limb-threatening arterial thrombi, we use our case to illustrate prompt restoration of blood flow via balloon catheter thrombectomy without complications. Balloon catheter thrombectomy is, in our opinion, superior to other treatment modalities in definitely restoring blood flow and preventing severe, even catastrophic, short-term and long-term limb complications. ${ }^{7-9}$ We recommend consideration of this approach when resources and personal are available for rapid intervention.

\section{Disclosures}

T.J.B.U., M.A.E., and T.R.L. have neither financial relationships nor conflicts of interest relevant to this article to disclose.

\section{References}

1 Mongale P, Chan A, Massicotte P, et al. Antithrombotic therapy in children, $9^{\text {th }}$ ed.: American College of Chest Physicians EvidenceBased Clinical Practice Guidelines. Chest 2012;141(2):e737S-e801S

2 Schmidt B, Andrew M. Neonatal thrombosis: report of a prospective Canadian and international registry. Pediatrics 1995;96(5 Pt 1): 939-943

3 Zetlitz E, Weiler-Mithoff E, Turner T. Idiopathic neonatal ischemia in the upper limb: the role of the microsurgeon. Am J Perinatol 2008;25(8):513-516

4 Saxonhouse MA, Burchfield DJ. The evaluation and management of postnatal thromboses. J Perinatol 2009;29(7):467-478

5 Andrew ME, Monagle P, deVeber G, Chan AKC. Thromboembolic disease and antithrombotic therapy in newborns. Hematology Am Soc Hematol Educ Program 2001;1:358-374

6 Robinson A, Fellows KE, Bridges ND, Rome JJ. Effectiveness of pharmacomechanical thrombolysis in infants and children. Am J Cardiol 2001;87(4):496-499, A8

7 Coombs CJ, Richardson PW, Dowling GJ, Johnstone BR, Monagle P. Brachial artery thrombosis in infants: an algorithm for limb salvage. Plast Reconstr Surg 2006;117(5):1481-1488

8 Lazarides MK, Georgiadis GS, Papas TT, Gardikis S, Maltezos C. Operative and nonoperative management of children aged 13 years or younger with arterial trauma of the extremities. J Vasc Surg 2006;43(1):72-76, discussion 76

9 Theile RJ, Coombs CJ. Microvascular and thrombolytic revascularization of an arm in a 32-week-gestation neonate: case report and review of the literature. J Reconstr Microsurg 1996;12(8):539-542

10 Weiner GM, Castle VP, DiPietro MA, Faix RG. Successful treatment of neonatal arterial thromboses with recombinant tissue plasminogen activator. J Pediatr 1998;133(1):133-136

11 Rashish G, Paes BA, Nagel K, Chan AK, Thomas S; Thrombosis and Hemostasis in Newborns (THiN) Group. Spontaneous neonatal arterial thromboembolism: infants at risk, diagnosis, treatment, and outcomes. Blood Coagul Fibrinolysis 2013;24(8):787-797

12 Long DK, Lorant DE. Multiple arterial thrombi and in utero leg gangrene in an infant of a diabetic mother. J Perinatol 2002;22(5): 424-427

13 Hsi AC, Davis DJ, Sherman FC. Neonatal gangrene in the newborn infant of a diabetic mother. J Pediatr Orthop 1985;5(3):358-360 